\title{
Life Style Management in Head and Neck Cancer Patients
}

\author{
M. K. Taneja
}

Received: 26 September 2013/Accepted: 8 October 2013/Published online: 19 November 2013

(C) Association of Otolaryngologists of India 2013

\begin{abstract}
Head and neck (HN) cancers involve highly visible body parts, structurally complex, and crucial to survival. A diagnosis of $\mathrm{HN}$ cancer, like any cancer diagnosis, is often accompanied by much fear and uncertainty. In addition, patients treated for $\mathrm{HN}$ cancer face difficulties in eating, chewing, drinking, breathing, speaking, as well as changes in appearance. Simultaneously, the burden of $\mathrm{HN}$ cancer is often manifested in psychosocial dysfunction, which can have a negative impact on quality of life (QL). Psychosocial interventions such as education along with cognitive-behavioral therapy generally provide an overall positive effect. With the growing impetus to investigate factors associated with these dysfunctions and disfigurement caused by the treatment, and considerable advancement in the development and validation of many global and disease-specific measures, there is an opportunity for further research to develop an appropriate clinical intervention program for such patients so that these patients can live a near normal life. Life style changes including dietary habits make gross changes in $\mathrm{QL}$.
\end{abstract}

Keywords Head and neck cancer - Quality of life . Psychosocial interventions - Life style management

\section{Introduction}

"The good physician treats the disease; the great physician treats the patient who has the disease."...Sir William Osler.

Many individuals have described a diagnosis of cancer as devastating, producing a more alarming response than

M. K. Taneja $(\square)$

E-982 C.R. Park, New Delhi, India

e-mail: ijo_editor@rediffmail.com that of any other disease process [1]. Newly diagnosed patients find themselves thrust on a journey that disorganizes their daily function, and is surrounded with much fear and uncertainty.

Patients with head and neck (HN) cancer have very specific and vast needs that are beyond the needs of most other patients diagnosed with other types of cancer. Frequently, many of these patients cannot conceal the effects of treatment because of the very visible nature of their condition and obvious functional difficulties. They must also embrace the reality of profound changes in relation to their speech, ability to swallow, taste, as well as noticeable alterations in appearance are more emotionally traumatic than any other type of cancer. Other undesirable side effects of treatment, such as poorly intelligible speech and drooling, can cause embarrassment and have a significant impact not only on social and family interactions but also on more internalized feelings of low self-esteem.

Despite significant investment in research to investigate different treatment regimens for $\mathrm{HN}$ cancer, limited improvement in patient survival has been achieved in the last 30 years in many countries [2]. One reason for this is that $\mathrm{HN}$ cancer is frequently diagnosed at an advanced stage. In addition to survival, assessment of quality of life (QL) issues after treatment has gained increased attention within cancer research. QL goes beyond physical and functional dimensions and what may be more important is how the patient with $\mathrm{HN}$ cancer functions within society after treatment [3, 4]. Herzon and Boshier [5] observed that patients placed immense importance on reentering society in a meaningful way and that this often precedes even fear of reoccurrence.

Hammerlid et al. [6] assessed prospectively the level of psychiatric morbidity and mental distress in newly diagnosed Swedish and Norwegian patients with HN cancer. 
Their findings highlighted that one-third of the participants were classified as possible/probable cases of major mood disorder using the Hospital Anxiety and Depression Scale (HADS) [7]. Such findings have been replicated in other studies, which demonstrate that anxiety and depression is experienced by approximately $30-40 \%$ of patients following treatment for $\mathrm{HN}$ cancer [8,9]. One outcome of depression is suicide, with Boulnd noting that patients with HN cancer form a relatively large proportion of suicide cases in cancer patients. This has also been acknowledged by Farberow et al. [10], who concluded that $2 \mathrm{HN}$ sites alone (tongue and pharynx) accounted for almost $20 \%$ of the total suicides among male patients with cancer.

Cognitive-behavioral therapy (CBT) has evolved with a robust evidence base, which makes it the currently preferred form of treatment for a wide range of anxiety and psychological disorders [11]. Researchers' use of CBT has demonstrated substantial benefits in cancer care [12], and in heterogeneous samples of patients with facial disfigurement [13]. These studies determined that psychological therapy based on the principles of CBT significantly reduced psychological morbidity $[12,13]$, providing justification for further investigation of the efficacy of cognitive behavioral interventions in patients with $\mathrm{HN}$ cancer.

\section{Head and Neck (HN) Cancer}

$\mathrm{HN}$ cancers include cancers of the lip, oral cavity, oropharynx, hypopharynx, tonsil, salivary glands, nasopharynx, nose, paranasal sinuses, and middle ear [14]. They are the sixth most common cancer worldwide [15]. Overall, 57.5\% of global $\mathrm{HN}$ cancers (excluding esophageal cancers) occur in Asia especially in India, for both sexes. HN cancers in India account for approximately $30 \%$ of all cancers in males and are nearly twice as common among men as they are among women. Over 200,000 cases of HN cancers occur each year in India [16]. There is a concern that the incidence is increasing, particularly in younger patients and in women. This shift in trend appears to result from changes in adult smoking prevalence, where the proportion of men who smoke has decreased and the proportion of young females who smoke has increased [17]. The majority of HN neoplasms are squamous cell carcinomas (approximately $80 \%$ ) [18]. The primary and most influential etiological factor for squamous cell carcinoma is tobacco use, with $80-90 \%$ of patients having a history of smoking. There is also substantial evidence to indicate that alcohol not only acts in synergy with tobacco to amplify the risk [19], but also is an independent risk factor. The addition of alcoholic drink, to intake of tobacco in any form further increases the risk for oral cancer by 11 -fold.

There are a number of other risk factors that have been postulated, including chronic candidal infection, nutritional and vitamin deficiency, and exposure to high levels of dust and chemicals [14]. Sexual behaviors are linked with cancer risk at the $\mathrm{HN}$ sites that have previously been associated with HPV infection. Having a history of six or more lifetime sexual partners and four or more lifetime oral sex partners has been linked with cancer of the oropharynx. Patients with dysplastic oral leukoplakia or melanoplakia have an average $30 \%$ risk of oral cancer. Plummer vinson syndrome characterized by iron deficiency anemia with deficiency in swallowing is another cause of cancer in non smokers which may be aggravated by tobacco and alcohol etc.

\section{The Challenge of HN Cancer for the Patient}

In HN cancer patients, complex care issues are best addressed by a multidisciplinary approach. Preservation of form and function are key elements in the overall management of HN cancer patients.

In a retrospective study on the incidence of side effects specifically related to eating in 25 patients with oropharyngeal cancer, $72 \%$ of patients required modified diets and $80 \%$ suffered from xerostomia for more than 1 year after curative treatment [20]. In addition, pain is often one of the most feared symptoms in cancer and can have a significant impact on both the QL of patients and their families.

\section{Quality of Life (QL)}

The term $Q L$ can be described as a person's perception of his/her ability to function in meaningful areas of living after illness as compared to before illness. QL is an integral part of assessment of outcomes in HN cancer.

Within the realm of cancer management, it is no longer satisfactory just to measure the effectiveness of treatment on survival alone, as cancer and its subsequent treatment may cause physical, emotional, and psychological difficulties for individuals. In other words, it is not only quantity of life that is important but also QL. Therefore, today the measurement of QL should be an adjunct to the traditional end points of overall survival, disease-free survival and tumor response in cancer management [21]. QL measurement should be routine, prospective and long term; using brief, patient-reported, validated tools, with both general and disease-specific modules. QL is positively correlated with the stage and site of the disease.

There is persuasive evidence to suggest that two lifestyle behaviors, physical activity (PA) and nutrition are necessary components in cancer survivorship programming [22]. PA has been shown to enhance patient functional capacity, physical functioning, and body composition, as well as facilitate the management of treatment related symptoms 
and side-effects including fatigue, and nausea [23, 24]. Diet rich in bitter almond, Curcuma longa, soy, mushroom extract, green tea extract should be given to cancer patients as these products are expected to prevent cancer recurrence.

Cancers of the oral cavity may need reconstructive and plastic surgery to rebuild bones or tissues. However, reconstructive surgery may not always be possible because of damage to the remaining tissue from the original surgery or from radiation therapy. If reconstructive surgery is not possible, a prosthodontist may be able to make a prosthesis (an artificial dental and/or facial part) and special training on how to use the device. Postoperatively in oral cancer patients, oral-motor and jaw exercises are initiated. Lingual, labial, and velar exercises are important in retraining speech articulation. Sensory stimulation and massage can be useful adjuncts to exercise in preventing fibrosis.

Swallowing strategies focus on safety (prevention of aspiration) and efficiency (ease and adequacy of oral intake). Common interventions include modifying food texture, head-positioning maneuvers (such as chin tuck to prevent laryngeal penetration or neck extension for gravity assist of oral transit), and behavioral techniques (doubleswallow or alternating textures to reduce pharyngeal residue; supraglottic swallow technique of breath-hold $\sim$ swallow $\sim$ cough sequence for aspiration risk). In some cases, adaptive equipment, such as long-handled utensils, syringe, or shortened straw (when suck is weak), facilitate oral intake. The strategies often require strict compliance and, therefore, intact cognition.

Patients' sense of self and personal motivation, as well as support from caregivers, is keys to recovery. Sensitivity and patience are necessary, and treating patients with personal care is essential. Staff must be aware that disfigured patients sitting in a crowded waiting room for lengthy periods of time may feel self-conscious and markedly uncomfortable. For some patients, communication may be clumsy (e.g., via a writing pad or a mechanical apparatus for laryngectomy patients) and time-consuming. Direct eye contact with the patient, without grimacing, is imperative.

There should be a social rehabilitation program (support team consisting of a ward nurse from a HN unit, psychologist, Speech and language therapist, Dental hygienist, Physiotherapist, Occupational therapist and a dietician) to promote coping in patients with $\mathrm{HN}$ cancer, managing psychosocial issues for patients with HN cancer by giving additional emotional support and information on HN cancer, its treatment, and side effects repeatedly after diagnosis. Greer et al. [25] has reported a gross improvement in QL after C.B.T.

Man is born with all inbuilt power to correct the body and fight illness. Most illness start in our minds and grow when abetted by fertilizer viz. depression, tobacco, alcohol, wrong food habits, environmental pollution and modulated by genes. Depression hostility and anger are the key factors regarding the fighting (immunity) power which is further aggravated by questionable drugs. There is a myth that cancer is genetic also. Gene alone cannot bring on any disease. Presence of a gene only indicates possibility. As human mind is the driving force for wellness as well for illness; hence psychotherapy, yoga, good psychosocial and physical environment not only prevent but lead to easy cure of cancer patients and their better rehabilitation.

\section{Life Style Management}

A person cannot control certain risk factors such as age, family history or race, but can limit other factors viz body weight, PA, diet, alcohol consumption, smoking or chewing tobacco, that affect the overall risk factor or recurrence of cancer.

Moderate exercise of 40-60 min 5 days a week can help prevent cancer development by increasing the body's disease fighting response. It has been seen that a 25 min brisk walk can reduce risk of cancer as much as $18 \%$. Deficiency of vitamin D may lead to early distant metastasis. Hence morning exercise in sun helps in prevention of cancer. Statistically significant higher rate of death has been reported among those who ate large amount of hydrogenated oils than who ate large amount of fruits, leafy vegetables, protein and omega-3 fatty acids. One should choose whole grain breads and cereals, drink $100 \%$ fruit or vegetable juices, make sure they are pasteurized. Limit sugary foods but try to eat frequently even if no appetite.

The meal of a cancer patient should be his favourite, appealing may be by adding lemon juice, cherry, tomatoes or other colourful garnishing.

Conquer constitution by drinking lot of water and eating high fiber foods like beans, lentils and leafy vegetables, orange juice has been shown to reduce the extent of DNA damage caused by cancer cells.

Protect mouth sores and avoid dry mouth by grinding or pureeing foods. Scrub and rinse the fruit and vegetables thoroughly to avoid diarrhea. Most of the cancer patients quit smoking but they have to remind passive smoking is more injurious to health. Even exposure to chemicals found in some work place, gasoline fumes and vehicle exhaust enhances the risk of cancer, and hence should be avoided.

Sour, sugarless hard candy or gum can stimulate saliva flow in patients having xerostomia. Patients should avoid mouth-drying substances such as caffeine, tobacco, and alcohol. Some patients try artificial saliva and mouthmoisturizing products and report relief.

Sleep is an important factor in wound healing. Warm almond milk, sleep time snacks may be a better choice than 
sleeping pills. Supplements of magnesium play a role in regulation of sleep. Always turn off your TV and all bedroom lights. Blue or night light inhibits melatonin secretion which disturbs sleep pattern. Illuminate with red light which is less disturbing. Morning exercise promotes restful sleep, body rhythms and release of growth hormones. Cooler body temperatures are associated with better sleep. Hence avoid late evening working or exercise. Bed room should be clean, no clutter. Put on comfortable loose clothes and extremely comfortable mattress with breathable linen.

\section{Conclusion}

Prevention and detection of HN cancer are best achieved through continued education of the public. Avoidance of alcohol and tobacco is stressed, and referral to supportive programs is provided. For many, lifestyle is altered once they are affected by cancer, and their lives can be fraught with personal and practical problems. Caring for patients with $\mathrm{HN}$ cancers requires the use of both the science and the art of social and symptom management. Interventions to maintain the airway, to provide nutritional support, to care for the skin, to help relearn swallowing and communicating, and to ensure adequate pain relief all are essential aspects to the successful management of this complex group of patients. Patients rely on the team to teach them what they must learn and to encourage them and their families through the difficult weeks and months of treatment. Patients and families need the team to teach and organize home care, whether the outcome is cure, disease control, progression, or death.

\section{References}

1. Sawyer H (2000) Meeting the information needs of cancer patients. Prof Nurse 15(4):244-247

2. Wadsworth JT, Somers KD, Stack BC et al (2004) Identification of patients with head and neck cancer using serum protein profiles. Arch Otolaryngol Head Neck Surg 130:98-104

3. Gritz ER, Carmack CL, de Moor C, Cossarelli A, Schacherer CW, Meyers EG (1999) First year after head and neck cancer: QL. J Clin Oncol 17(1):352-362

4. Argerakis GP (1990) Psychosocial considerations of the posttreatment of head and neck cancer patients. Dent Clin North Am 34(2):285-305
5. Herzon F, Boshier M (1990) Head and neck cancer-emotional management. Head Neck Surg 2:112-118

6. Hammerlid E, Persson L, Sullivan M, Westin T (1999) Qualityof-life effects of psychosocial intervention in patients with head and neck cancer. Otolaryngol Head Neck Surg 120:507-516

7. Zigmond AS, Snaith RP (1983) The Hospital Anxiety and Depression Scale. Acta Psychiatr Scand 63:361-370

8. Morton RP, Davies ADM, Baker J, Baker GA, Stell PM (1984) QL in treated head and neck cancer patients: a preliminary report. Clin Otolaryngol 9:181-185

9. Boulnd G (1985) Suicide and cancer, II: medical factors in suicide by cancer patients in Sweden, 1973-1976. J Psychosoc Oncol 3:31-35

10. Farberow NL, Ganzler S, Cutter F, Reynolds D (1971) An eightyear survey of hospital suicides. Life Threat Behav 1(3):184-202

11. Marks IM (2000) Forty years of psychosocial treatments. Behav Cogn Psychother 28:323-334

12. Moorey S, Greer S, Watson M (1994) Adjuvant psychological therapy for patients with cancer. Psycho-Oncology 7:218-228

13. Newell R, Clarke M (2000) Evaluation of a self-help leaflet in treatment of social difficulties following facial disfigurements. Int J Nurs Stud 37:381-388

14. Davis R (1998) Nursing management of head and neck cancer. Macmillan Nurse 7:7-14

15. Kamangar F, Dores GM, Anderson WF (2006) Patterns of cancer incidence, mortality, and prevalence across five continents: defining priorities to reduce cancer disparities in different geographic regions of the world. J Clin Oncol 24(14):2137-2150

16. Kulkarni MR (2013) Head and neck cancer burden in India. Int J Head and Neck Surg 4(1):29-35

17. Department of Health, Social Services and Public Health (DHSSPS) (2003) A five-year tobacco action plan 2003-2008. DHSSPS, Belfast, Northern, Ireland

18. Eakin R (2001) Head and neck cancer. In: Spence RAJ, Johnston PG (eds) Oncology. Oxford University Press, Oxford, pp 191-208

19. Craddock C (1993) Head and neck cancer prevention: the new challenge. Semin Oncol Nurs 9(3):169-173

20. Beeken L, Calman F (1994) A return to normal eating after curative treatment for oral cancer. What are the long-term prospects? Eur J Cancer Oral Oncol 30(B):387-392

21. Bowling A (2001) Measuring disease, 2nd edn. Open University Press, Buckingham

22. Thomas R, Davies N (2007) Lifestyle during and after cancer treatment. Clin Oncol (R Coll Radiol) 19(8):616-627

23. McNeely M, Peddle C, Parliament M, Courneya K (2006) Cancer rehabilitation: recommendations for integrating exercise programming in the clinical practice setting. Curr Cancer Ther Rev 2(4):35

24. van Weert E, Hoekstra-Weebers J, Otter R, Postema K, Sanderman R, van der Schans C (2006) Cancer-related fatigue: predictors and effects of rehabilitation. Oncologist 11(2):184-196

25. Greer S, Moorey S, Baruch JDR et al (1992) Adjuvant psychological therapy for patients with cancer: a prospective randomised trial. Br Med J 304:675-680 